\title{
Are we strong enough to assert our rights in quality healthcare?
}

\section{Loris Brunetta}

\author{
Thalassaemia International Federation (TIF); Associazione Ligure Thalassemici Onlus, Italy
}

\begin{abstract}
The title of this speech is an important challenge for me, for a patient I mean, to face because it's not easy to state today if we are really strong enough to assert our rights for a quality healthcare.

At first sight, and in an optimistic vision, we could answer to this question YES, we are, but I think we need to explore better the field before to confirm that this is the right answer to the question.

The first thing to assess is what we mean with the pronoun WE: the patients and parents' community represented from TIF? The whole community that plays around thalassemia and hemoglobinopathies, meaning patients and parents and scientists? What else?
\end{abstract}

\section{Are we strong enough to assert our rights in quality healthcare?}

TIF is a fantastic synthesis of our presence at every level today, it's big enough to represent with successful the patients, as actually does, but needs to move forward, trying to become more productive and more conscious of the great role that patients can play in the future, it's time to have faith in their capacities.

The scientific community acts every day in a context really complicated from the scarce resources they have available for moving forward in quality healthcare, but despite this difficulties it achieved extraordinary results and the outcomes are better where there is a patients/parents' association that works closely with the scientists.

With any evidence we know very well what we need to move forward in the field of hemoglobinopathies and for us what we will try to achieve in the near future is what we mean for "quality healthcare. And, in my opinion, this is a common and concrete necessity, therefore we should need to move forward together.

Correspondence: Loris Brunetta, Thalassaemia International Federation (TIF); Associazione Ligure Thalassemici Onlus, Italy

E-mail: lorisbrunetta@gmail.com

Key words: hemoglobinopathies; healthcare; thalassemia; Thalassemia International Federation; TIF.

(C) Copyright L. Brunetta, 2014

Licensee PAGEPress, Italy

Thalassemia Reports 2014; 4:4882

doi:10.4081/thal.2014.4882

This article is distributed under the terms of the Creative Commons Attribution Noncommercial License (by-nc 3.0) which permits any noncommercial use, distribution, and reproduction in any medium, provided the original author(s) and source are credited.
As you know, I am a patient and so I will try to explore the perspective, from a patient's point of view. It needs to be considered from two separate fields: the scientific and the social field.

The scientific field requires particular attention to the new developments in the clinical treatment, the new frontier of Gene Therapy but also some problems that are not anymore emerging but now consolidated, as osteoporosis and pain that are reflecting in worsening the quality of patients' life, some heavy problems as the fight against the C Hepatitis and the new frontiers of the treatment for this disease. Give to our patients the possibility to have an easy access to the best treatments worldwide should be our first goal to achieve as soon as possible, trying to overcome the differences from country to country.

No less important is the social field especially in times of economic crisis where the difficult situation that many countries are living put at high risk to maintain and to respect many rights that the patients achieved in the last decades, through many strong efforts by our associations.

Furthermore the immigration flow is bringing an increased number of people suffering from thalassemia and, above all, sickle cell disease, that require to have an adequate treatment. This is happening in some areas of Europe, never touched before, so the mapping of the needs is changing very quickly and we should be ready to face this new emergency.

The economic crisis is the more visible problem, but it's not the only one we have to face. It's clear that many problems cannot be addressed by few countries but they need to be governed by the European Institutions that, unfortunately, seem to be not fitted to face the challenges of the present times. As representatives of patients, we need to become more productive in our action at institutional level, but the European institutions need to be more effective and quick in the decision making process.

For facing all these important issues we need to have strong leaderships both in scientific and in social field and a close cooperation between the two parts so to cope with the process putting on the carpet all our best resources and able to provide to the European institutions all our support.

The very recent Manifesto presented from TIF in this Conference goes in right direction in my opinion, really congratulations to TIF's President Mr. Englezos for this, is exactly what we mean today for close cooperation of all the resources that plays around thalassemia and hemoglobinopathies.

But beyond the words we need to put on the field an effective strong willing to work together in the awareness that joining the resources, always respecting the different roles, it's the only chance we have for moving forward or for defending what we have achieved in the last decades.

Without any doubt the leading role of some European countries in the field of hemoglobinopathies, like Italy and UK, must be the guide through this dramatic period to maintain the excellence of our thalassemia centers all across the Europe and to achieve more important results in the future of clinical research for the benefit of all our patients.

The excellent assistance provided in many European centers, especially in Italy, Cyprus, Greece and UK, is pivotal for the patients. Being 
treated into a specialized center is quite different than being treated outside it, in terms of lifespan, therefore in terms of quality of life.

It's necessary to have clear in our mind a vision on the future, an open mind for facing with creativity and consciousness the problems we are living, a pool of expert patients' very involved in the work at high level with the institutions.

Times are changing very fast, if some time ago the patients played simply a passive role into healthcare, today they can represent a strong voice and a qualified support in the framework of public health.

There are many resolutions of European Union that try to give to the patients a more significant active role in the decision making process, but the procedures are often too complicated for convincing the patients to adhere to these programs. It's pivotal for the institutions to facilitate the access to the patients, showing to trust on them.

To the patients I have to say that for to consolidate this new status, and for being credible we need to be organized and to evaluate with particular attention every step forward in order to avoid dangerous misunderstandings.

We must understand that the real challenge today is played at European level on the field of Rare Disease. Joining together into a strong European context is important for strengthen our action and ensuring our patients that we are monitoring very closely what happens.

Being at this level means to have access to the funds that the European Community provides for high level projects, in the field of therapy and at social level, means to monitor carefully all the procedures for the registration of new drugs, avoiding dangerous delay, means to have visibility and voice where the decision making process happens, and much more.

Of course for working at such a level is necessary to promote an alliance between the patients and the scientists, with respect of the role that each one has to play, so to have stronger voice.
For promoting this alliance is extremely urgent to come back to work closer than in the past, by recognizing that the developments in the scientific field are also due to the hard work done by the patients and parents' Associations all along the last decades.

'Till this moment I have continuously referred to the patients because I trust that really we can be the bosses of our future but we cannot forget the impressive work and the strong willingness of our parents and the role that many of them are still playing in our society.

It's time to go back to share opinions and to accept each other visions, in order to give a new and more modern impulse to our action.

Forbidding to the patients the access to the scientific sessions at the conferences, even the more experts amongst them, therefore, it's not the best thing to do. We need a new and modern vision of the patient, not anymore as an object of study and a consumer of drugs but as a subject carrying rights that have to be respected.

Even Thalassemia International Federation, has to change partially its vision, finding the courage to invest much more on the patients, to trust them much more than ever done in the past, to promote their involvement at highest level.

For doing a productive work together, the patients need to be prepared and educated; Thalassemia International Federation has prepared program for building the capacity of Associations' members and for educating patients to become experts, this event is an example of that, we need to join TIF that is putting in action every possible effort to unite all the patients worldwide.

Let's go back to the question now! When and if all these issues will be put into the same logical frame we can answer to the question: YES, now we are strong enough for assert our rights. It would be a terrible mistake to consider us on the finishing line, because up to now we have still some road in front of us to run but we are on the right way. 